\title{
Ruído em hospital universitário: impacto na qualidade de vida
}

\author{
Noise in the hospital setting: impact on quality of life
}

\author{
Monique Cantelli da Silva', Vivian Baptista da Luz', Daniela Gil²
}

\section{RESUMO}

Objetivo: Aferir os níveis de ruído em distintos ambientes de um hospital universitário e investigar o impacto dessa exposição na qualidade de vida dos profissionais que atuam nesses ambientes. Métodos: $\mathrm{O}$ ruído foi aferido por meio de medidor de pressão sonora em três períodos do dia: manhã, tarde e noite, durante uma semana, em diferentes dependências de um hospital, a saber: UTI neonatal, nutrição, anfiteatros, gráfica, lavanderia, marcenaria e serralheria. Para cinco trabalhadores de cada setor, foi solicitado o preenchimento dos questionários de hábitos auditivos e de Qualidade de vida (WHOQOL-Bref), adaptado para o Português Brasileiro. Para cada questão do WHOQOL-Bref atribuiu-se de 0 a 5 pontos, sendo que, ao final, quanto maior a pontuação, melhor qualidade de vida o indivíduo considera ter. Resultados: Houve diferença significativa entre os ambientes, para os valores mínimos e máximos de pressão sonora, independentemente do período do dia em que foi realizada a aferição. Todos os ambientes puderam ser considerados prejudiciais. Nos escores apresentados nas questões de qualidade de vida geral e nos domínios do WHOQOL-Bref (Qualidade de Vida, Físico, Psicológico, Relações Sociais e Meio Ambiente), observou-se que não houve diferença entre os setores. Conclusão: Os níveis mínimos e máximos de ruído em todos os setores, com exceção da UTI neonatal, excedem os previstos para ambiente hospitalar; a variação dos níveis de ruído caracteriza ambientes com ruídos intermitentes que colocam em risco a saúde auditiva dos funcionários; os níveis de ruído aferidos evidenciam a necessidade de implementação de Programa de Conservação Auditiva, com medidas coletivas e individuais.

Descritores: Perda auditiva provocada por ruído; Ruído ocupacional; Ambiente de instituições de saúde; Ambiente de trabalho; Qualidade de vida

\begin{abstract}
Purpose: To determine noise levels in different hospital settings and investigate the impact of noise exposure on the quality of life of the healthcare workers in these environments. Methods: Noise was measured using a sound level meter in different sectors of the Sao Paulo university hospital (neonatal intensive care unit, nutrition, classrooms, print shop, laundry, carpentry shop and metalwork shop) in different periods of the day (morning, afternoon and night) over a one-week period. Five workers from each sector were asked to fill out questionnaires on hearing habits and quality of life (WHOQoL-Bref), adapted to Brazilian Portuguese. Each question on the WHOQoL-Bref was scored from 0 to 5 points, with higher overall scores denoting better quality of life. Results: Statistically significant differences among environments were found regarding minimum and maximum sound levels, regardless of the time of the day. All sound levels were considered harmful. No statistically significant differences among sectors were found in the overall quality of life score or subscales of the WHOQoL-Bref (Quality of Life, Physical Aspects, Psychological Aspects, Social Relations and Environment). Conclusion: The minimum and maximum noise levels in all sectors, except the neonatal intensive care unit, exceeded those defined for hospital environments. The variation in noise levels characterizes environments with intermittent noise that places the auditory health of the employees at risk. The noise levels encountered demonstrate the need for a hearing conservation program involving both group and individual measures.
\end{abstract}

Keywords: Hearing loss, noise-induced; Noise, occupational; Health facility environment; Working environment; Quality of life

Trabalho realizado no Hospital São Paulo, Hospital Universitário, Universidade Federal de São Paulo - UNIFESP - São Paulo (SP), Brasil, com auxílio da Fundação de Amparo à Pesquisa do Estado de São Paulo (FAPESP), bolsa de Iniciação Científica.

(1) Programa de Pós-graduação (Mestrado) em Distúrbios da Comunicação Humana, Universidade Federal de São Paulo - UNIFESP - São Paulo (SP), Brasil.

(2) Departamento de Fonoaudiologia, Universidade Federal de São Paulo - UNIFESP - São Paulo (SP), Brasil.

Conflito de interesses: Não

Contribuição dos autores: $M C S$ : pesquisador principal, elaboração da pesquisa, elaboração do cronograma, levantamento da literatura, coleta, análise dos dados, redação do artigo, submissão e trâmites do artigo; $V B L$ : pesquisador auxiliar, elaboração da pesquisa, coleta e redação do artigo; $D G$ : orientador, elaboração da pesquisa, elaboração do cronograma, análise dos dados, correção da redação do artigo, aprovação da versão final.

Endereço para correspondência: Monique Cantelli da Silva. R. Botucatu, 802, Vila Clementino, São Paulo (SP), Brasil, CEP: $04023-062$.

E-mail: moniquecantelli@gmail.com

Recebido em: 15/2/2012; Aceito em: 14/5/2013 


\section{INTRODUÇÃO}

Segundo a norma regulamentadora (NR) 15 , quando a exposição a ruído intenso é continuada, em média $85 \mathrm{~dB}(\mathrm{~A})$ por oito horas diárias, podem ocorrer alterações estruturais e funcionais na orelha interna, que determinam a ocorrência da Perda Auditiva Induzida por Nível de Pressão Sonora Elevado (PAINPSE), sendo que a cada $5 \mathrm{~dB}(\mathrm{~A})$ de acréscimo (a partir de $85 \mathrm{~dB}$ ), o tempo de exposição deve ser reduzido pela metade ${ }^{(1)}$.

Segundo a Associação Brasileira de Normas Técnicas (ABNT), os níveis de conforto acústico variam de 35 a 55 $\mathrm{dB}(\mathrm{A})$ para diferentes ambientes hospitalares ${ }^{(2)}$.

A partir de 1989, a Organização Mundial da Saúde (OMS) passou a tratar o ruído como problema de saúde pública, recomendando, em 1993, que o nível de ruído nos hospitais seja de até $40 \mathrm{~dB}(\mathrm{~A})$ durante o dia e $35 \mathrm{~dB}(\mathrm{~A})$ durante a noite ${ }^{(3)}$.

Sintomas auditivos da PAINPSE alteram e diminuem a qualidade de vida do indivíduo, tanto no âmbito laboral, como no social e no familiar. Além disso, sabe-se que a exposição ao ruído pode levar a sintomas extra-auditivos ${ }^{(4)}$.

Visto que a PAINPSE pode ser acompanhada de transtornos que comprometerão a qualidade de vida do trabalhador, a conscientização e a prevenção são medidas necessárias a serem tomadas antes que se instale a perda auditiva.
Assim, o objetivo deste estudo foi aferir os níveis de ruído em distintos ambientes de um hospital universitário e investigar o impacto dessa exposição na qualidade de vida dos profissionais que atuam nesses ambientes.

\section{MÉTODOS}

O projeto desta pesquisa foi aprovado pelo Comitê de Ética em Pesquisa da Universidade Federal de São Paulo (UNIFESP), sob o número 1975/08. Os participantes voluntários concordaram em assinar o Termo de Consentimento Livre e Esclarecido, atendendo às Normas para a Realização de Pesquisa em Seres Humanos, Resolução 196/96, do Conselho Nacional de Saúde, de 10 de outubro de 1996. Trata-se de um estudo transversal descritivo, realizado em sete setores do Hospital São Paulo, com funcionários expostos a ruídos, contínuos ou intermitentes, produzidos pelo maquinário do setor durante as horas diárias trabalhadas. Foram selecionadas as seguintes áreas: UTI neonatal, nutrição, anfiteatros, gráfica, lavanderia, marcenaria e serralheria, a partir dos resultados de um trabalho anteriormente realizado em um dos ambientes e por apresentarem níveis elevados de ruído(4).

Os setores, o maquinário e os turnos de trabalho estão apresentados no Quadro 1.

Quadro 1. Setores hospitalares, seus respectivos maquinários e turnos de trabalho

\begin{tabular}{|c|c|c|c|}
\hline Setor & \multicolumn{2}{|c|}{ Maquinário } & Turnos \\
\hline UTI Neonatal & $\begin{array}{l}\text { - } 30 \text { incubadoras (algumas de fototerapia) } \\
\text { - dispositivos de aviso } \\
\text { - monitores de sinais vitais }\end{array}$ & $\begin{array}{l}\text { - oxímetros } \\
\text { - campainha de porta } \\
\text { - geladeira } \\
\text { - equipe de profissionais }\end{array}$ & Das $7 \mathrm{~h}$ às $17 \mathrm{~h}$ e das $17 \mathrm{~h}$ às $7 \mathrm{~h}$ \\
\hline Nutrição & $\begin{array}{l}\text { - exaustores } \\
\text { - fogão } \\
\text { - talheres e louças } \\
\text { - panelas }\end{array}$ & $\begin{array}{l}\text { - liquidificadores } \\
\text { - batedeiras } \\
\text { - equipe de funcionários }\end{array}$ & Das $7 \mathrm{~h}$ às $16 \mathrm{~h}$ \\
\hline Anfiteatros & $\begin{array}{l}\text { - telefone } \\
\text { - televisor } \\
\text { - computador }\end{array}$ & $\begin{array}{l}\text { - alunos } \\
\text { - professores } \\
\text { - equipe de funcionários }\end{array}$ & Das $8 \mathrm{~h}$ às $22 \mathrm{~h}$ \\
\hline Gráfica & $\begin{array}{l}\text { - impressoras } \\
\text { - offset } \\
\text { - guilhotina } \\
\text { - diversos maquinários }\end{array}$ & $\begin{array}{l}\text { - televisor } \\
\text { - campainha de porta } \\
\text { - telefone } \\
\text { - equipe de funcionários }\end{array}$ & Das $9 \mathrm{~h}$ às $16 \mathrm{~h}$ \\
\hline Marcenaria & $\begin{array}{l}\text { - martelo } \\
\text { - serrote } \\
\text { - serras } \\
\text { - lixadeira } \\
\text { - furadeira } \\
\text { - esquadria }\end{array}$ & $\begin{array}{l}\text { - pinadores } \\
\text { - tupia } \\
\text { - diversos maquinários } \\
\text { - rádio } \\
\text { - equipe de funcionários }\end{array}$ & Das $7 \mathrm{~h}$ às $16 \mathrm{~h}$ \\
\hline Serralheria & $\begin{array}{l}\text { - serra elétrica } \\
\text { - máquina de solda } \\
\text { - lixadeira }\end{array}$ & $\begin{array}{l}\text { - ferramentas } \\
\text { - rádio } \\
\text { - equipe de funcionários }\end{array}$ & Das $7 \mathrm{~h}$ às $16 \mathrm{~h}$ \\
\hline
\end{tabular}


Aos supervisores de cada setor foi solicitado que informassem quais os períodos de maior volume de trabalho e, consequentemente, de maior nível de ruído (dia da semana, período do dia: manhã, tarde ou noite, turnos dos funcionários, quantidade em horas de maquinário ativo) para programação da aferição do ruído ambiental. Além disso, foi obtida a devida autorização dos supervisores para aferir os níveis de pressão sonora e aplicação de questionários para os funcionários dos respectivos setores.

A partir do relato dos supervisores, as medições foram assim programadas: pela manhã (entre às $7 \mathrm{~h}$ e $8 \mathrm{~h}$ ), à tarde (entre às $12 \mathrm{~h}$ e às $13 \mathrm{~h}$ ) e à noite (entre às $17 \mathrm{~h}$ e às $18 \mathrm{~h}$ ).

As medições dos níveis de pressão sonora foram realizadas com medidor de pressão sonora modelo RadioShack Digital Sound-Level Meter, aferido conforme especificação do fabricante.

A medição foi realizada utilizando o circuito de compensação "A" e resposta lenta (slow), conforme recomendação da norma regulamentadora NR 15 - Anexo 1, da Portaria 3.214/78 ${ }^{(1)}$.

Cada medição durou cinco minutos, sendo mantido o mesmo posicionamento da pesquisadora (ao centro do setor) a cada aferição. Durante o período da medição, foi considerado o valor mínimo como sendo a intensidade mais fraca e o valor máximo como sendo a intensidade mais forte de pressão sonora aferida em cada setor, obtendo-se, assim o nível de pressão sonora equivalente (Leq).

Observou-se também o uso de Equipamento de Proteção Individual (EPI) para proteção auditiva, pelos funcionários, em cada ambiente em questão.

Para cinco trabalhadores voluntários de cada setor, foi solicitado o preenchimento do questionário de hábitos auditivos adaptado $^{(5)}$ (Anexo 1) para obter dados sobre sua história clínica e do questionário de Qualidade de vida WHOQOL-Bref, adaptado para o Português Brasileiro( ${ }^{(6)}$ (Anexo 2).

Os questionários foram apresentados aos funcionários, devendo ser respondidos e entregues à avaliadora em dia previamente combinado, mediante a assinatura do Termo de Consentimento Livre e Esclarecido. Ambos os questionários foram respondidos sem o auxílio e/ou a interferência da examinadora.

O primeiro questionário, destinado a analisar os hábitos auditivos dos trabalhadores, foi composto por perguntas referentes à exposição a ruído ocupacional e não ocupacional, sendo dividido em seis partes: identificação, dados ocupacionais, história de perda auditiva na família, hábitos auditivos, história otológica e medicamentos utilizados. As questões foram objetivas, tendo havido exceção com relação à questão referente aos nomes dos medicamentos utilizados pelo entrevistado ${ }^{(5)}$.

O segundo questionário, que investigou a qualidade de vida geral dos trabalhadores expostos a ruído, foi desenvolvido pela Organização Mundial da Saúde (OMS) e é denominado World Health Organization Quality of Life (WHOQOL-Bref), versão abreviada do WHOQOL-100 ${ }^{(7)}$. Utilizou-se a versão abreviada do questionário, traduzido e validado para o Português Brasileiro $^{(6)}$, conhecido como Avaliação da Qualidade de Vida. É composto por 26 questões sobre qualidade de vida, com cinco variações possíveis de respostas quanto ao grau de satisfação. As respostas são organizadas em cinco classes (Quadro 2). O indivíduo foi instruído a responder o questionário considerando as duas semanas anteriores ao preenchimento.

Das 26 questões do WHOQOL-Bref, duas são gerais e as demais representam cada uma das 24 facetas que compõem o instrumento original. Assim, diferentemente do WHOQOL-100 em que cada uma das 24 facetas é avaliada a partir de quatro questões, no WHOQOL-Bref as facetas são avaliadas por apenas uma questão. Tal questionário foi pontuado atribuindo-se 0 a 5 pontos por questão, sendo que quanto maior a pontuação, melhor qualidade de vida o indivíduo considera ter. A partir dessa pontuação, a qualidade de vida pôde ser classificada em escalas de avaliação, capacidade e frequência, como observado no Quadro $2^{(6)}$.

As respostas obtidas no WHOQOL-Bref foram analisadas por meio de testes estatísticos, visando comparar os dados obtidos no questionário WHOQOL-Bref (entre os seus domínios e entre os domínios e os setores de trabalho) e os níveis de ruído em cada setor (nos diferentes períodos e nos diferentes ambientes de trabalho).

Foram utilizados os testes estatísticos de Mann-Whitney, teste de Kruskal Wallis e Intervalo de Confiança para a Média. Foi utilizado o software aplicativo do tipo científico Statistical Package for the Social Sciences (SPSS) V16 para pontuação dos escores do questionário WHOQOL-Bref, pois não é aconselhada a pontuação manual, uma vez que pode aumentar a margem de erros no cálculo dos escores ${ }^{(6,7)}$.

Definiu-se um nível de significância de $0,05(5 \%)$ e todos os intervalos de confiança, ao longo do trabalho, foram construídos

Quadro 2. Escala de respostas para as escalas de Avaliação, Capacidade e Frequência

\begin{tabular}{|lccccc|}
\hline Escala & $0 \%$ (âncora) & $25 \%$ & $50 \%$ & $75 \%$ & $100 \%$ (âncora) \\
\hline \multirow{3}{*}{ Avaliação } & Muito insatisfeito & Insatisfeito & Nem satisfeito nem insatisfeito & Satisfeito & Muito satisfeito \\
& Muito ruim & Ruim & Nem ruim nem bom & Bom & Muito bom \\
& Muito infeliz & Infeliz & Nem feliz nem infeliz & Feliz & Muito feliz \\
\hline Capacidade & Nada & Muito pouco & Médio & Muito & Completamente \\
\hline Frequência & Nunca & Raramente & Às vezes & Repetidamente & Sempre \\
\hline
\end{tabular}

Fonte: OMS, 1998. Versão em português dos instrumentos de avaliação de qualidade de vida (WHOQOL). Disponível em: http://www.ufrgs.br/psiq/whoqol3.html 
com $95 \%$ de confiança estatística.

Os resultados com significância estatística foram destacados com o símbolo asterisco (*) e os resultados com tendência a serem significantes com o símbolo sustenido (\#). Quando não foi possível utilizar a estatística, utilizou-se a marcação -x.

\section{RESULTADOS}

As Tabelas 1, 2 e 3 mostram as medidas descritivas da comparação dos níveis de pressão sonora aferidos nos setores hospitalares, em cada um dos períodos do dia (manhã, tarde e noite).
Houve diferença entre os ambientes hospitalares tanto para os valores mínimos quanto para os máximos, independentemente do período do dia em que foi realizada a aferição (Tabelas 1, 2 e 3 ).

Abaixo, estão os resultados obtidos, considerando-se os quatro domínios que compreendem o questionário. Vale ressaltar que as questões 1 e 2 são consideradas como um domínio à parte, denominado Qualidade de Vida. Neste estudo, as respostas variaram entre $25 \%$ e $49 \%$ para todos os setores, o que indicou a classificação da qualidade de vida geral dos indivíduos como insatisfatória e ruim.

Na Tabela 4 estão apresentadas as medidas descritivas e

Tabela 1. Medidas descritivas dos níveis mínimo e máximo de ruído aferidos no período da manhã nos diferentes ambientes do Hospital São Paulo

\begin{tabular}{|c|c|c|c|c|c|c|c|}
\hline Manhã & & Média & Mediana & $\mathrm{DP}$ & $\mathrm{n}$ & IC & Valor de $p$ \\
\hline \multirow{7}{*}{ Mínimo } & Anfiteatro & 57,2 & 56,0 & 4,4 & 5 & 3,9 & \multirow{7}{*}{$<0,001^{*}$} \\
\hline & Gráfica & 57,8 & 58,0 & 6,9 & 5 & 6,1 & \\
\hline & Lavanderia & 84,2 & 84,0 & 1,5 & 5 & 1,3 & \\
\hline & Marcenaria & 68,8 & 64,0 & 9,1 & 5 & 8,0 & \\
\hline & Nutrição & 76,8 & 77,0 & 0,8 & 5 & 0,7 & \\
\hline & Serralheria & 66,4 & 65,0 & 4,3 & 5 & 3,7 & \\
\hline & UTI & 59,6 & 60,0 & 3,8 & 5 & 3,4 & \\
\hline \multirow{7}{*}{ Máximo } & Anfiteatro & 60,4 & 59,0 & 3,8 & 5 & 3,3 & \multirow{7}{*}{$0,002^{*}$} \\
\hline & Gráfica & 64,6 & 69,0 & 7,0 & 5 & 6,2 & \\
\hline & Lavanderia & 89,0 & 89,0 & 1,2 & 5 & 1,1 & \\
\hline & Marcenaria & 81,4 & 73,0 & 16,9 & 5 & 14,8 & \\
\hline & Nutrição & 84,2 & 84,0 & 3,3 & 5 & 2,9 & \\
\hline & Serralheria & 82,8 & 70,0 & 20,8 & 5 & 18,2 & \\
\hline & UTI & 68,2 & 68,0 & 2,9 & 5 & 2,5 & \\
\hline
\end{tabular}

*Valores significativos ( $\mathrm{p} \geq 0,05)$ - Teste Mann-Whitney

Legenda: DP = desvio-padrão; IC = intervalo de confiança; UTI = unidade de terapia intensiva

Tabela 2. Medidas descritivas dos níveis mínimo e máximo de ruído aferidos no período da tarde nos diferentes ambientes do Hospital São Paulo

\begin{tabular}{|c|c|c|c|c|c|c|c|}
\hline Tarde & & Média & Mediana & DP & $\mathrm{n}$ & IC & Valor de $p$ \\
\hline \multirow{7}{*}{ Mínimo } & Anfiteatro & 63,4 & 61,0 & 9,0 & 5 & 7,9 & \multirow{7}{*}{$0,001^{*}$} \\
\hline & Gráfica & 60,0 & 60,0 & 8,0 & 5 & 7,0 & \\
\hline & Lavanderia & 84,4 & 85,0 & 3,1 & 5 & 2,7 & \\
\hline & Marcenaria & 62,4 & 62,0 & 1,7 & 5 & 1,5 & \\
\hline & Nutrição & 76,8 & 77,0 & 0,8 & 5 & 0,7 & \\
\hline & Serralheria & 66,6 & 63,0 & 8,3 & 5 & 7,2 & \\
\hline & UTI & 59,0 & 58,0 & 3,9 & 5 & 3,4 & \\
\hline \multirow{7}{*}{ Máximo } & Anfiteatro & 72,2 & 75,0 & 6,8 & 5 & 6,0 & \multirow{7}{*}{$0,001^{*}$} \\
\hline & Gráfica & 66,2 & 65,0 & 4,8 & 5 & 4,2 & \\
\hline & Lavanderia & 90,2 & 90,0 & 2,9 & 5 & 2,5 & \\
\hline & Marcenaria & 70,2 & 70,0 & 2,0 & 5 & 1,8 & \\
\hline & Nutrição & 81,2 & 81,0 & 1,9 & 5 & 1,7 & \\
\hline & Serralheria & 82,4 & 77,0 & 11,7 & 5 & 10,3 & \\
\hline & UTI & 69,2 & 69,0 & 3,3 & 5 & 2,9 & \\
\hline
\end{tabular}

*Valores significativos ( $\mathrm{p} \geq 0,05)$ - Teste Mann-Whitney

Legenda: DP = desvio-padrão; IC = intervalo de confiança; UTI = unidade de terapia intensiva 
Tabela 3. Medidas descritivas dos níveis mínimo e máximo de ruído aferidos no período da noite nos diferentes ambientes do Hospital São Paulo

\begin{tabular}{|c|c|c|c|c|c|c|c|}
\hline Noite & & Média & Mediana & DP & $n$ & IC & Valor de $p$ \\
\hline \multirow{7}{*}{ Mínimo } & Anfiteatro & 64,6 & 68,0 & 7,8 & 5 & 6,9 & \multirow{7}{*}{$0,001^{*}$} \\
\hline & Gráfica & 75,2 & 81,0 & 9,3 & 5 & 8,2 & \\
\hline & Lavanderia & 84,4 & 84,0 & 1,1 & 5 & 1,0 & \\
\hline & Marcenaria & $-x-$ & $-x-$ & $-x-$ & $-x-$ & $-x-$ & \\
\hline & Nutrição & 75,8 & 76,0 & 1,9 & 5 & 1,7 & \\
\hline & Serralheria & $-x-$ & $-x-$ & $-x-$ & $-x-$ & $-x-$ & \\
\hline & UTI & 54,8 & 55,0 & 4,0 & 5 & 3,5 & \\
\hline \multirow{7}{*}{ Máximo } & Anfiteatro & 71,4 & 73,0 & 4,8 & 5 & 4,2 & \multirow{7}{*}{$0,001^{*}$} \\
\hline & Gráfica & 79,8 & 83,0 & 7,8 & 5 & 6,8 & \\
\hline & Lavanderia & 89,2 & 89,0 & 1,3 & 5 & 1,1 & \\
\hline & Marcenaria & $-x-$ & $-x-$ & $-x-$ & $-x-$ & $-x-$ & \\
\hline & Nutrição & 81,2 & 81,0 & 1,9 & 5 & 1,7 & \\
\hline & Serralheria & $-x-$ & $-x-$ & $-x-$ & $-x-$ & $-x-$ & \\
\hline & UTI & 68,4 & 68,0 & 3,6 & 5 & 3,2 & \\
\hline
\end{tabular}

*Valores significativos $(p \geq 0,05)$ - Teste Mann-Whitney

Legenda: DP = desvio-padrão; IC = intervalo de confiança; UTI = unidade de terapia intensiva

Tabela 4. Medidas descritivas para o domínio Físico e Psicológico do WHOQOL-Bref nos diferentes setores do Hospital São Paulo

\begin{tabular}{|c|c|c|c|c|c|c|c|c|c|c|c|c|c|c|}
\hline & $\begin{array}{l}\text { Lav. } \\
\text { fís }\end{array}$ & $\begin{array}{c}\text { Lav. } \\
\text { psi }\end{array}$ & $\begin{array}{l}\text { Nut. } \\
\text { fís }\end{array}$ & $\begin{array}{l}\text { Nut. } \\
\text { psi }\end{array}$ & $\begin{array}{l}\text { Marc. } \\
\text { fís }\end{array}$ & $\begin{array}{c}\text { Marc. } \\
\text { psi }\end{array}$ & $\begin{array}{l}\text { Ser. } \\
\text { fís }\end{array}$ & $\begin{array}{l}\text { Ser. } \\
\text { psi }\end{array}$ & $\begin{array}{l}\text { Anf. } \\
\text { fís }\end{array}$ & $\begin{array}{l}\text { Anf. } \\
\text { psi }\end{array}$ & $\begin{array}{c}\text { Gráf. } \\
\text { fís }\end{array}$ & $\begin{array}{c}\text { Gráf. } \\
\text { psi }\end{array}$ & $\begin{array}{l}\text { UTI } \\
\text { fís }\end{array}$ & $\begin{array}{l}\text { UTI } \\
\text { psi }\end{array}$ \\
\hline Média & 66,4 & 63,3 & 65,0 & 59,2 & 72,9 & 63,3 & 80,0 & 62,5 & 79,5 & 65,0 & 69,3 & 60,0 & 80,0 & 60,8 \\
\hline Mediana & 64,3 & 62,5 & 71,4 & 62,5 & 71,4 & 62,5 & 89,3 & 62,5 & 82,1 & 66,7 & 67,9 & 58,3 & 78,6 & 62,5 \\
\hline $\mathrm{DP}$ & 18,1 & 16,8 & 20,1 & 8,0 & 10,6 & 11,9 & 16,3 & 6,6 & 7,0 & 2,3 & 17,8 & 7,0 & 16,3 & 12,0 \\
\hline $\mathrm{n}$ & 5 & 5 & 5 & 5 & 5 & 5 & 5 & 5 & 5 & 5 & 5 & 5 & 5 & 5 \\
\hline IC & 15,9 & 14,7 & 17,6 & 7,0 & 9,3 & 10,5 & 14,3 & 5,8 & 6,1 & 2,0 & 15,6 & 61 & 14,3 & 10,5 \\
\hline Valor de $p$ & 0,633 & 0,893 & 0,633 & 0,893 & 0,633 & 0,893 & 0,633 & 0,893 & 0,633 & 0,893 & 0,633 & 0,893 & 0,633 & 0,893 \\
\hline
\end{tabular}

Teste Kruskal-Wallis $(p \geq 0,05)$

Legenda: Lav. = lavanderia; Nut. = nutrição; Marc. = marcenaria; Ser. = serralheria; Anf. = anfiteatros; Gráf. = gráfica; UTI = unidade de terapia intensiva; Fís. = domínio físico; Psi. = domínio psicológico; DP = desvio-padrão; IC = intervalo de confiança

Tabela 5. Medidas descritivas para o domínio Social e Meio Ambiente do WHOQOL-Bref nos diferentes setores do Hospital São Paulo

\begin{tabular}{|c|c|c|c|c|c|c|c|c|c|c|c|c|c|c|}
\hline & $\begin{array}{l}\text { Lav. } \\
\text { soc }\end{array}$ & $\begin{array}{l}\text { Lav. } \\
\text { amb }\end{array}$ & $\begin{array}{l}\text { Nut. } \\
\text { soc }\end{array}$ & $\begin{array}{l}\text { Nut. } \\
\text { amb }\end{array}$ & $\begin{array}{c}\text { Marc. } \\
\text { soc }\end{array}$ & $\begin{array}{c}\text { Marc. } \\
\text { amb }\end{array}$ & $\begin{array}{l}\text { Ser. } \\
\text { soc }\end{array}$ & $\begin{array}{l}\text { Ser. } \\
\text { amb }\end{array}$ & $\begin{array}{l}\text { Anf. } \\
\text { soc }\end{array}$ & $\begin{array}{l}\text { Anf. } \\
\text { amb }\end{array}$ & $\begin{array}{l}\text { Gráf. } \\
\text { soc }\end{array}$ & $\begin{array}{l}\text { Gráf. } \\
\text { amb }\end{array}$ & $\begin{array}{l}\text { UTI } \\
\text { soc }\end{array}$ & $\begin{array}{l}\text { UTI } \\
\text { amb }\end{array}$ \\
\hline Média & 73,3 & 53,8 & 53,3 & 46,3 & 83,3 & 46,9 & 86,7 & 56,3 & 78,3 & 52,4 & 75,0 & 48,1 & 75,0 & 61,9 \\
\hline Mediana & 75,0 & 50,0 & 58,3 & 40,6 & 91,7 & 43,8 & 91,7 & 59,4 & 83,3 & 50,0 & 75,0 & 43,8 & 75,0 & 59,4 \\
\hline DP & 17,1 & 14,9 & 40,7 & 18,3 & 19,5 & 11,0 & 11,2 & 7,7 & 17,3 & 11,6 & 13,2 & 12,2 & 15,6 & 9,7 \\
\hline$n$ & 5 & 5 & 5 & 5 & 5 & 5 & 5 & 5 & 5 & 5 & 5 & 5 & 5 & 5 \\
\hline IC & 15,0 & 13,1 & 35,6 & 16,0 & 17,1 & 9,7 & 9,8 & 6,7 & 15,1 & 10,2 & 11,6 & 10,7 & 13,7 & 8,5 \\
\hline Valor de $p$ & 0,622 & 0,290 & 0,622 & 0,290 & 0,622 & 0,290 & 0,622 & 0,290 & 0,622 & 0,290 & 0,622 & 0,290 & 0,622 & 0,290 \\
\hline
\end{tabular}

Teste Kruskal-Wallis $(\mathrm{p} \geq 0,05)$

Legenda: Lav. = lavanderia; Nut. = nutrição; Marc. = marcenaria; Ser. = serralheria; Anf. = anfiteatros; Gráf. = gráfica; UTI = unidade de terapia intensiva; Soc. = domínio relações sociais; Amb. = domínio meio ambiente; DP = desvio-padrão; IC = intervalo de confiança

obtidas no domínio Físico e Psicológico do WHOQOL-Bref.

Na Tabela 5 estão apresentadas as medidas descritivas e obtidas no domínio Relações Sociais e Meio Ambiente do WHOQOL-Bref.

Observou-se que nos domínios do questionário (Tabelas 4 e 5), as diferenças encontradas entre os grupos não puderam ser consideradas significativas, ou seja, os funcionários dos diferentes setores apresentaram qualidade de vida semelhante, quando medida por um questionário geral.

\section{DISCUSSÃO}

Os níveis máximos de ruído aferidos nos diferentes setores demonstraram que os setores de lavanderia, marcenaria, 
serralheria, gráfica e nutrição são de risco para desenvolvimento de PAINPSE, sendo prejudiciais caso esses níveis de ruído sejam contínuos, uma vez que, pela legislação (Anexo 1 da NR 15), quando um indivíduo é exposto a um nível de ruído igual ou superior a $85 \mathrm{~dB}$ por 8 horas, potencialmente pode colocar em risco sua saúde auditiva.

Pode-se observar que os valores médios dos níveis mínimos e máximos de ruído aferidos nos três períodos do dia nos diferentes setores do Hospital São Paulo, (HSP) ultrapassaram, em todos os ambientes, os níveis máximos recomendados pela ABNT e OMS. No entanto, ao analisar os níveis mínimos de ruído nas mesmas tabelas, verificou-se que também se mostraram mais elevados que os recomendados pela ABNT (NBR $10152)^{(2)}$, cuja norma afirma que níveis aceitáveis de ruído em diferentes ambientes hospitalares devem estar entre 35 e 55 $\mathrm{dB}(\mathrm{A})$, sendo que os valores obtidos neste estudo foram superiores, classificando esses ambientes como ruidosos (entre 60 e $70 \mathrm{~dB}$ ). Em todos os setores, com exceção da UTI neonatal, os valores mínimos ultrapassaram a faixa de classificação de ambiente ruidoso. Alguns, inclusive, excederam os valores propostos pela NR 15, como na lavanderia. Esses achados corroboram com a literatura pesquisada ${ }^{(8)}$.

Sendo o ruído prejudicial para a audição e para a qualidade de vida do ser humano, a $\mathrm{OMS}^{(9)}$ recomendou que, nos hospitais, o nível de ruído seja de até $40 \mathrm{~dB}$ (A) durante o dia e $35 \mathrm{~dB}(\mathrm{~A})$ durante o período noturno. Todavia, os valores obtidos em todos os setores do Hospital São Paulo transpõem esses níveis nos distintos horários. No entanto, a diferença estatística entre os ambientes é esperada, pois são ambientes com estruturas e rotinas de trabalho extremamente diferentes.

Os níveis máximos aferidos nas dependências hospitalares do presente estudo assemelham-se aos das empresas metalúrgicas e/ou indústrias, devido aos elevados níveis de pressão sonora $^{(10)}$. Entretanto, devido ao método de medição, não é possível realizar a comparação direta entre os ambientes hospitalares deste estudo e as indústrias.

Pode-se observar que em cada setor houve uma expressiva variação na média dos valores mínimos e máximos de ruído: anfiteatro $\rightarrow 57,2$ a 72,2 dB; gráfica $\rightarrow 57,8$ a 79,8 dB; lavanderia $\rightarrow 84,2$ a $90,2 \mathrm{~dB}$; marcenaria $\rightarrow 62,4$ a $81,4 \mathrm{~dB}$; nutrição $\rightarrow 75,8$ a $84,2 \mathrm{~dB}$; serralheria $\rightarrow 66,4$ a $82,8 \mathrm{~dB}$; UTI neonatal $\rightarrow 54,8$ a $69,2 \mathrm{~dB}$.

Apesar dos ambientes do presente estudo não envolverem a frequência de pacientes, os profissionais que atuam nesses ambientes estão sujeitos aos níveis de pressão sonora elevados.

A UTI neonatal apresentou o menor nível de pressão sonora, supostamente pela precaução tomada em relação aos bebês para que não acordem e pelo uso de instrumentos menos ruidosos quando comparados aos dos demais ambientes do hospital envolvidos neste estudo, ressaltando que há legislação específica para o setor da UTI, mencionando os cuidados particulares que se fazem necessários ${ }^{(4,11,12)}$.

Apesar dos níveis de ruído da UTI neonatal estarem dentro dos valores recomendados pela legislação específica, a literatura já quantificou que há grande intensidade de ruído na manipulação de incubadoras ou nos finais de semana. Em alguns estudos, houve a necessidade de intervenção nas rotinas e na conduta dos profissionais visando a saúde auditiva dos trabalhadores e dos pacientes ${ }^{(11,12)}$.

Sendo assim, é imprescindível a implementação de um Programa de Conservação Auditiva (PCA) no Hospital São Paulo para que os profissionais sejam beneficiados na sua função laborativa, com a redução efetiva da poluição sonora, como prevê a legislação pela norma regulamentadora (NR-9) ${ }^{(1)}$.

A importância desses programas já foi discutida na literatura, fortalecendo, assim, a ideia a partir dos resultados apresentados neste trabalho, mostrando que a realização de exames audiométricos periódicos permite o acompanhamento da saúde auditiva dos trabalhadores, identificando o desencadeamento ou progressão de uma perda auditiva. Além disso, deve-se elaborar e realizar orientações sobre prevenção e conservação auditivas (medidas de proteção coletivas e individuais), apresentação de diferentes EPIs, entre outros ${ }^{(4,13,14)}$.

Pode-se observar os resultados positivos do investimento em PCAs voltados para o controle da emissão de ruídos na fonte, pois os programas objetivam não apenas a manutenção da saúde auditiva, mas também a diminuição dos acidentes de trabalho ${ }^{(15)}$.

Segundo alguns estudos, a eficácia dos treinamentos que fazem parte do PCA foi avaliada em relação a outro grupo que não recebeu esse treinamento, deixando clara a importância do investimento em ações educativas. A partir dos resultados desses estudos, tornou-se clara a necessidade do uso de estratégias educacionais e de motivação adequadas para o uso efetivo de $\mathrm{EPI}^{(16,17)}$.

Apesar de haver fornecimento de EPI para os empregados do Hospital São Paulo pelo Serviço de Segurança e Medicina do Trabalho (SESMT), não há treinamento adequado ou controle de uso e guarda dos EPIs, o que é fundamental para o aproveitamento apropriado e proteção, como comprovado na literatura $^{(4,8,14-17)}$. Embora não tenha sido realizado um levantamento específico em relação aos EPIs, foi possível observar que a maioria dos funcionários não os utilizava em sua jornada de trabalho.

Em relação à Qualidade de Vida geral, mesmo não havendo diferenças entre os setores, todos julgam ter uma qualidade de vida regular, o que pode, entre outras coisas, refletir um ambiente de trabalho insalubre. Pelo fato do preenchimento do questionário ter sido voluntário, pode-se inferir que os indivíduos que o completaram poderiam representar os extremos, ou seja, ótima qualidade de vida ou péssima qualidade de vida e, por isso, desejaram manifestar-se.

Para o domínio Físico, os setores da lavanderia, nutrição, marcenaria e gráfica apresentaram resultados na faixa de 50\% a 74\%, enquanto que os setores da serralheria, anfiteatros e UTI neonatal, na faixa de $75 \%$ a $99 \%$, indicando que a qualidade 
de vida dos funcionários da serralheria, anfiteatros e UTI neonatal, com relação ao domínio físico, foi melhor avaliada do que nos demais setores hospitalares estudados. Vale lembrar que a lavanderia foi o setor considerado como o mais ruidoso, permitindo inferir que esse aspecto poderia ter influenciado o julgamento quanto à qualidade de vida no domínio físico. $\mathrm{O}$ ruído pode causar disfunção cognitiva de uma variedade de mecanismos. Há efeitos do ruído descritos sobre o processo de sono que também podem contribuir para o comprometimento de tarefas cognitivas e desempenho global ${ }^{(18)}$.

Para o domínio Psicológico, todos os setores apresentaram resultados na faixa de $50 \%$ a $74 \%$, isto é, a maioria das respostas foi "nem satisfeito, nem insatisfeito", "nem ruim, nem bom", "mais ou menos", "médio" e "frequentemente". Estudos mostraram que o ruído é um fator preocupante e perturbador em UTIs e que pode afetar a frequência cardíaca e pressão arterial de pacientes ${ }^{(19,20)}$. Outra hipótese é que o ruído cause um elevado nível de excitação, resultando na incapacidade de concentração ${ }^{(18)}$. Esses efeitos extra-auditivos psicológicos e fisiológicos consequentes do ruído são prejudiciais à saúde e podem comprometer a qualidade de vida.

Para o domínio Relações Sociais, os setores da lavanderia e nutrição estavam na faixa de $50 \%$ a $74 \%$, enquanto que os setores da marcenaria, serralheria, anfiteatros, gráfica e UTI neonatal, na faixa de $75 \%$ a $99 \%$, isto é, a maioria das respostas foi "satisfeito", "bom", "bastante", "muito" e "muito frequentemente". O resultado apontou que para os setores da marcenaria, serralheria, anfiteatros, gráfica e UTI neonatal, a qualidade de vida quanto às relações sociais é melhor do que a dos setores da lavanderia e da nutrição. Indivíduos sensíveis ao ruído podem atrasar o término da resposta simpática devido a um desacoplamento do sistema nervoso autônomo e dos circuitos pré-frontais que interpretam estímulos estressantes e enviam a resposta adequada ao estresse apropriado $^{(21)}$. Como resultado, ocorre o aborrecimento do indivíduo e o acúmulo de estresse, podendo, então, apresentar-se de modo negativo nas relações pessoais com demais funcionários e/ou companheiros.

Para o domínio Meio Ambiente, os setores da nutrição, marcenaria e gráfica estavam na faixa de $25 \%$ a $49 \%$, enquanto que os setores da lavanderia, serralheria, anfiteatros e UTI neonatal, na faixa de $50 \%$ a $74 \%$.

Os resultados obtidos nos diferentes domínios do questionário foram semelhantes, talvez por não estarem relacionados exclusivamente à exposição ao ruído, sofrendo influência de outras morbidades que cada indivíduo tenha apresentado, em particular. Há relatos na literatura que, entre as morbidades comuns relacionadas aos trabalhadores expostos ao ruído, estão a hipertensão arterial, as doenças respiratórias, estresse, zumbido e tontura, queixas relatadas durante o preenchimento do questionário de hábitos auditivos, podendo interferir nos aspectos psicossociais do indivíduo ${ }^{(5,22-26)}$.

Muitos profissionais da área da saúde, entre eles enfermeiros, médicos e fisioterapeutas, percebem os danos do ruído, concordando com os relatos dos funcionários durante a aplicação dos questionários ${ }^{(27)}$. Tem havido poucas pesquisas sobre as influências das condições acústicas na equipe de saúde. Embora os pacientes sejam o centro de atenção de todos os hospitais, o grau de satisfação dos funcionários com o ambiente de trabalho afeta diretamente a eficiência das tarefas, o entusiasmo e a qualidade dos cuidados prestados ${ }^{(19)}$.

Como instruído anteriormente à aplicação dos questionários, o indivíduo deveria basear-se em suas experiências nas duas últimas semanas. Desta forma, os dados obtidos podem ter sofrido influência dessas vivências individuais. Sendo assim, a qualidade de vida poderia estar mais próxima da adequação naquele momento, não necessariamente se mantendo do mesmo modo ao longo do tempo, como também observado na literatura ${ }^{(26)}$.

Pode-se observar que o setor da nutrição apresentou, na maioria das vezes, o menor escore nos domínios do WHOQOLBref, ou seja, uma qualidade de vida ruim, além de ser o segundo setor com maior intensidade de ruído.

Os domínios Meio Ambiente e Qualidade de Vida apresentaram os escores mais baixos entre os domínios do WHOQOLBref, o primeiro podendo ser justificado pelas condições do local onde o indivíduo mora, meios de transporte, acesso aos serviços de saúde, geralmente criticados pela população como um todo. O segundo domínio refere-se a como o indivíduo está enxergando-se naquele momento com relação a sua qualidade de vida e sua saúde, o que pode também interferir nos aspectos psicológicos, como se observa no domínio Psicológico, com apresentação de baixos escores nos setores da nutrição, gráfica e UTI neonatal. Por outro lado, o domínio Relações Sociais mostrou escores mais altos, indicando que esses indivíduos encontram em seu ambiente de trabalho a melhor forma possível para cumprir suas obrigações e exercê-las de maneira equilibrada em seu dia a dia.

Cabe ressaltar que o objetivo do presente estudo foi avaliar o impacto do ruído na qualidade de vida em contextos diferenciados, além do laborale, por isso, foi escolhido um questionário de qualidade de vida geral. Esse questionário foi pouco utilizado no Brasil, sobretudo relacionado a questões auditivas.

Atualmente, existe um questionário de qualidade de vida no trabalho, o Quality of Working Life Questionnaire - Questionário de Qualidade de Vida no Trabalho (QWLQ$78)^{(28)}$, que poderia revelar dados mais específicos do que os encontrados no presente estudo. No entanto, esse instrumento não pode ser utilizado, uma vez que estava em elaboração.

Apesar disso, a comparação entre os resultados no escore global do QWLQ-78 e o QWLQ-Bref não destoou em mais do que cinco pontos percentuais em todos os domínios, sendo que o primeiro apenas demanda menos tempo de aplicação que o último ${ }^{(29)}$.

Apesar dos achados terem permitido algumas conclusões importantes, há necessidade de aumentar o número de voluntários para preenchimento dos questionários, bem como de 
utilizar um instrumento mais específico, como o QWLQ-78, e, principalmente, utilizar testes audiológicos visando melhor documentar o impacto dos níveis de ruído na qualidade de vida desses funcionários.

\section{CONCLUSÃO}

A partir da aferição dos níveis de ruído em diferentes ambientes do Hospital São Paulo e da análise das respostas do WHOQOL-Bref, concluiu-se que: os níveis mínimos e máximos de ruído em todos os setores, com exceção da UTI neonatal, excedem os previstos na legislação específica para ambiente hospitalar; a variação dos níveis de ruído caracteriza ambientes com ruídos intermitentes que colocam em risco a saúde auditiva; os níveis de ruído aferidos evidenciam a necessidade de implementação de Programa de Conservação Auditiva (PCA), com medidas coletivas e individuais, visando impedir o desencadeamento e/ou agravamento de PAINPSE; a qualidade de vida é semelhante entre os domínios do questionário e entre os setores, tendo sido classificada entre regular e boa pela maioria, com exceção dos domínios Qualidade de Vida e Meio Ambiente, que foram classificados como ruins.

\section{REFERÊNCIAS}

1. Brasil. Ministério do Trabalho e Emprego. Portaria $n^{\circ} 3.214$, de 08 de junho de 1978. Aprova as Normas Regulamentadoras - NR - do Capítulo V, Título II, da Consolidação das Leis do Trabalho, relativas à Segurança e Medicina do Trabalho. Diário Oficial da República Federativa do Brasil, Brasília, DF, 6 jul. 1978.

2. Associação Brasileira de Normas Técnicas (ABNT). NBR 10152. Níveis de ruído para conforto acústico procedimento. Rio de Janeiro: Associação Brasileira de Normas Técnicas, 1987.

3. Fernandes JC. Acústica e ruídos. UNESP - Faculdade de Engenharia - Bauru, Departamento de Engenharia Mecânica, Laboratório de Acústica e Vibrações - LAV, set. 2005. [citado 2008 fev 05]. Disponível em: http://wwwp.feb.unesp.br/jcandido/acustica/apostila. htm.

4. Silva MC, Orlandi CG, Chang EM, Armellini PFS, Pinto MM, Gil D, et al. Níveis de ruído na lavanderia de um hospital público. Rev CEFAC 2011;13:472-8.

5. Hanazumi A, Gil D. Hábitos auditivos dos alunos de graduação e especialização de Fonoaudiologia da UNIFESP [monografia]. São Paulo: Universidade Federal de São Paulo; 2008.

6. Departamento de Psiquiatria e Medicina Legal da Faculdade de Medicina da UFRGS. Divisão de Saúde Mental. Grupo WHOQOL. Versão em português dos instrumentos de avaliação de qualidade de vida (WHOQOL). 1998. [citado 2008 jul 22]. Disponível em: http:// www.ufrgs.br/psiquiatria/psiq/whoqoll.html

7. Fleck MPA, Leal OF, Louzada S, Xavier M, Chachamovich E, Vieira $\mathrm{G}$, et al. Desenvolvimento da versão em português do instrumento de avaliação de qualidade de vida da Organização Mundial da Saúde (WHOQOL-100). Rev Bras Psiquiatr. 1999;21(1):19-28.
8. Otenio MH, Cremer E, Claro EMT. Intensidade de ruído em hospital de 222 leitos na $18^{\text {a }}$ Regional de Saúde - PR. Rev Bras Otorrinolaringol. 2007;73(2):245-50.

9. International Programme on chemical Safety. Environmental Health Criteria 12. Noise. Geneva, Switzerland: World Health Organization; 1980. Disponível em: http://www.inchem.org/documents/ehc/ehc/ ehc012.htm. ISBN 9241540729.

10. Guerra MR, Lourenço PMC, Bustamante-Teixeira MT, Alves MJM. Prevalência de perda auditiva induzida por ruído em empresa metalúrgica. Rev Saúde Pública. 2005;39(2):238-44.

11. Rodarte MDO, Scochi CGS, Leite AM, Fujinaga CI, Zamberlan NE, Castral TC. O ruído gerado durante a manipulação das incubadoras: implicações para o cuidado de enfermagem. Rev Latino-Am Enfermagem. 2005;13(1):79-85.

12. Kakehashi TY, Pinheiro EM, Pizzarro G, Guilherme A. Nível de ruído em unidade de terapia intensiva neonatal. Acta Paul Enferm. 2007;20(4):404-9.

13. Gonçalves CGO, Iguti AM. Análise de programas de preservação da audição em quatro indústrias metalúrgicas de Piracicaba, São Paulo, Brasil. Cad Saúde Pública. 2006;22(3):609-18.

14. El Dib RP, Atallah ÁN, Andriolo RB, Soares BGO, Verbeek J. A systematic review of the interventions to promote the wearing of hearing protection. São Paulo Med J. 2007;125(6):359-61.

15. Dias A, Cordeiro R, Gonçalves CGO. Exposição ocupacional ao ruído e acidentes do trabalho. Cad Saúde Pública. 2006;22(10):2125-30.

16. Bramatti L, Morata TC, Marques JM. Ações educativas com enfoque positivo em programa de conservação auditiva e sua avaliação. Rev CEFAC. 2008;10(3):398-408.

17. Faria CAR, Suzuki FA. Avaliação dos limiares auditivos com e sem equipamento de proteção individual. Rev Bras Otorrinolaringol. 2008;74(3):417-22.

18. Seidman MD, Standring RT. Noise and quality of life. Int J Environ Res Public Health. 2010;7(10):3730-8.

19. Xie H, Kang J, Mills GH. Clinical review: The impact of noise on patients' sleep and the effectiveness of noise reduction strategies in intensive care units. Crit Care. 2009;13(2):208.

20. Suh MH, Wen JK, Wen CL, Sheng JH, Robert JC, Chung YL, et al. Associations of exposure to noise with physiological and psychological outcomes among post-cardiac surgery patients in ICUs. Clinics (São Paulo). 2010;65(10):985-9.

21. Shepherd D, Welch D, Dirks KN, Mathews R. Exploring the relationship between noise sensitivity, annoyance and health-related quality of life in a sample of adults exposed to environmental noise. Int J Environ Res Public Health. 2010;7(10):3579-94.

22. Fernandes M, Morata TC. Estudo dos efeitos auditivos e extraauditivos da exposição ocupacional a ruído e vibração. Rev Bras Otorrinolaringol. 2002;68(5):705-13.

23. Marchiori LLM, Rego Filho EA, Matsuo T. Hipertensão como fator associado à perda auditiva. Rev Bras Otorrinolaringol. 2006;72(4):533-40.

24. Ogido R, Costa EA, Machado HC. Prevalência de sintomas auditivos e vestibulares em trabalhadores expostos a ruído ocupacional. Rev Saúde Pública. 2009;43(2):377-80. 
25. Prestes R, Gil D. Impact of tinnitus on quality of life, loudness/ pitch match and high frequency audiometry. Int Tinnitus J. 2009;15(2):134-8.

26. Seligman J. Efeitos não-auditivos e aspectos psicossomáticos no indivíduo submetido a ruído intenso. Rev Bras Otorrinolaringol. 1993;59(4):257-9.

27. Jardini L, Pires MPO. O ruído na unidade de terapia intensiva pediátrica: opiniões de profissionais de enfermagem de alguns hospitais de São Paulo [monografia]. São Paulo: Universidade
Federal de São Paulo; 2007.

28. Reis Júnior DR. Qualidade de vida no trabalho: construção e validação do questionário QWLQ-78 [dissertação]. Ponta Grossa: Universidade Tecnológica Federal do Paraná; 2008.

29. Cheremeta M, Pedroso B, Pilatti LA, Kovaleski JL. Construção da versão abreviada do QWLQ-78: um instrumento de avaliação da qualidade de vida no trabalho. Rev Bras Qualidade de Vida. 2011;3(1):1-15.

Anexo 1. Questionário de hábitos auditivos

Questionário - Audiologia Clínica (Áudio Ocupacional) - Adaptado(5)

1. Identificação:

Nome:

Idade:

Sexo:

Endereço:

Telefone:

Grau de escolaridade:

Profissão:

Repouso auditivo (> 14 horas):

Data da avaliação:

1.1 Raça: Branca ( ) Negra ( ) Pardo ( ) Amarela ( )

1.2 Preferência manual: Direita ( ) Esquerda ( )

2. Dados ocupacionais

Empresa onde trabalha: Ramo de atividade:

Setor: Função: Horas diárias:

Tempo de trabalho na empresa:

Descrição das atividades e do ambiente de trabalho:

Exposição a produtos químicos (solventes, metais, etc): ( ) Não

Exposição à vibração: ( ) Não

( ) $\operatorname{Sim}$

Tempo:

Exposição a níveis elevados de pressão sonora (ruído etc): ( ) Não

Impressão sobre o ruído: ( ) Baixo

Uso de Protetor Auricular: ( ) Não

( ) Médio ( ) Alto

( ) Sim $\rightarrow$ Concha ( )

Utiliza durante toda a jornada de trabalho? ( ) Não

Exposição anterior a ruído: Ramo:

Função:
( ) Sim Tempo:

( ) Sim Tempo:

Plug ( ) Outros ( )

Tempo:

\section{Tem história de perda auditiva na família? Qual o grau de parentesco?}

\section{Hábitos auditivos}

a. Costuma ouvir música? Sim ( ) Não( )

- Que tipo de música? Pop ( ) Rock ( ) Samba ( ) Axé ( ) Pagode ( ) Outros

- Quantas horas por dia? Menos de 2 horas ( ) Entre 2 a 3 horas ( ) Entre 3 a 4 horas ( ) entre 4 a 5 horas ( ) Mais que 5 horas ( )

- Com que tipo de aparelho? Aparelho de som ( ) Mp3 player/ iPod ( ) Outros

- Usa fones de ouvido? Sim ( ) Não ( )

- De qual tipo? Sobre a orelha ( ) Dentro do canal auditivo ( )

- Costuma ouvir música fora de casa? ( ) Sim ( ) Não

- Metrô ( ) Carro ( ) Ônibus ( )

- Quanto tempo por dia? Menos de 30 minutos ( ) Entre 30 minutos e 1 hora ( )

Entre 1 hora e 2 horas ( ) Mais de 2 horas ( )

b. Pratica esporte? Sim ( ) Não( )

- Qual(is)?

\section{História otológica}

a. Cirurgias anteriores: ouvidos ( ) nariz ( ) garganta ( )

b. Dores de ouvido ( )

c. Otorreia ( )

d. Zumbido ( ) Há quanto tempo? 
e. Características do zumbido

- Descrição: agudo ( ) grave ( ) alto ( ) médio ( ) baixo ( ) não sabe ()

- Grau de severidade: leve ( ) moderado ( ) severo ( )

- OE ( ) OD ( ) Bilateral ( )

- Qual a frequência que você nota zumbido em sua cabeça ou ouvidos?

Nunca ( ) aproximadamente uma vez toda semana ( ) aproximadamente uma vez em poucos dias [menos de 1 semana] ( )

Uma vez ou mais todo dia ( ) a todo momento ( )

- Em que período é mais intenso: dia inteiro ( ) manhã ( ) tarde ( ) noite ( ) mais de um período ( )

- Quanto esses ruídos preocupam ou te perturbam?

Não é aplicável a mim ( ) Não me preocupam ( ) Me preocupam um pouco ( ) Me preocupam moderadamente ( ) Me preocupam muito ( )

f. Tontura ( )

- Descrição: rotatória ( ) não rotatória ( ) postural ( ) não postural ( )

- Sintomas associados: náuseas ( ) alterações visuais( ) desmaios ( )

Taquicardia ( ) outros

- Quando não está sob influência de álcool ou drogas, com que frequência você sofre ataques de tontura, instabilidade?

Nunca ( ) Pelo menos uma vez toda semana ( ) Pelo menos uma vez em poucos dias [menos de 1 semana] ( )

Uma vez ou mais todo dia ( ) Todo o tempo ( )

- Quanto à tontura ou a instabilidade, preocupam ou te perturbam?

Não é aplicável a mim ( ) Não me preocupam ( ) Me preocupam um pouco ( ) Me preocupam moderadamente ( ) Me preocupam muito ( ) g. Trauma craniano ou acidente ( )

\section{Toma medicamentos? Para quê?}

Anexo 2. Questionário de qualidade de vida - Questionário WHOQOL-Bref adaptado (OMS, 1998)

Instruções: Este questionário é sobre como você se sente a respeito de sua qualidade de vida, saúde e outras áreas de sua vida. Por favor, responda a todas as questões. Se você não tem certeza sobre que resposta dar em uma questão, por favor, escolha entre as alternativas a que Ihe parece mais apropriada. Esta, muitas vezes, poderá ser sua primeira escolha. Por favor, tenha em mente seus valores, aspirações, prazeres e preocupações. Nós estamos perguntando o que você acha de sua vida, tomando como referência as duas últimas semanas. Por exemplo, pensando nas últimas duas semanas, uma questão poderia ser:

\begin{tabular}{|l|c|c|c|c|c|}
\hline & Nada & $\begin{array}{c}\text { Muito } \\
\text { pouco }\end{array}$ & Médio & Muito & Completamente \\
\hline Você recebe dos outros o apoio de que necessita? & 1 & 2 & 3 & 4 & 5 \\
\hline
\end{tabular}

Você deve circular o número que melhor corresponde ao quanto você recebeu dos outros o apoio de que necessitou nestas últimas duas semanas. Portanto, você deve circular o número 4 se você recebeu "muito" apoio, como acima.

\begin{tabular}{|l|c|c|c|c|c|}
\hline & Nada & $\begin{array}{c}\text { Muito } \\
\text { pouco }\end{array}$ & Médio & Muito & Completamente \\
\hline Você recebe dos outros o apoio de que necessita? & 1 & 2 & 3 & 4 & 5 \\
\hline
\end{tabular}

Você deve circular o número 1 se você não recebeu "nada" de apoio.

Por favor, leia cada questão, veja o que você acha e circule o número que lhe parece ser a melhor resposta.

\begin{tabular}{|l|l|c|c|c|c|c|}
\hline & & Muito ruim & Ruim & $\begin{array}{c}\text { Nem ruim, nem } \\
\text { boa }\end{array}$ & Boa & Muito boa \\
\hline 1 & Como você avaliaria sua qualidade de vida? & 1 & 2 & 3 & 4 & 5 \\
\hline & & $\begin{array}{c}\text { Muito } \\
\text { insatisfeito }\end{array}$ & Insatisfeito & $\begin{array}{c}\text { Nem satisfeito } \\
\text { nem insatisfeito }\end{array}$ & Satisfeito & Muito satisfeito \\
\hline 2 & Quão satisfeito (a) você está com a sua saúde? & 1 & 2 & 3 & 4 & 5 \\
\hline
\end{tabular}

As questões seguintes são sobre o quanto você tem sentido algumas coisas nas últimas duas semanas.

\begin{tabular}{|l|l|c|c|c|c|}
\hline & & Nada & $\begin{array}{c}\text { Muito } \\
\text { pouco }\end{array}$ & Mais ou menos & Bastante \\
\hline 3 & $\begin{array}{l}\text { Em que medida você acha que sua dor (física) } \\
\text { impede você de fazer o que você precisa? }\end{array}$ & 1 & 2 & 3 & 4 \\
\hline 4 & $\begin{array}{l}\text { O quanto você precisa de algum tratamento médico } \\
\text { para levar sua vida diária? }\end{array}$ & 1 & 2 & 3 & 4 \\
\hline 5 & O quanto você aproveita a vida? & 1 & 2 & 3 & 4 \\
\hline 6 & $\begin{array}{l}\text { Em que medida você acha que a sua vida tem } \\
\text { sentido? }\end{array}$ & 1 & 2 & 3 & 5 \\
\hline
\end{tabular}




\begin{tabular}{|l|l|c|c|c|c|c|}
\hline 7 & O quanto você consegue se concentrar? & 1 & 2 & 3 & 4 & 5 \\
\hline 8 & Quão seguro(a) você se sente em sua vida diária? & 1 & 2 & 3 & 4 & 5 \\
\hline 9 & $\begin{array}{l}\text { Quão saudável é o seu ambiente físico (clima, } \\
\text { barulho, poluição, atrativos)? }\end{array}$ & 1 & 2 & 3 & 4 \\
\hline
\end{tabular}

As questões seguintes perguntam sobre quão completamente você tem sentido ou é capaz de fazer certas coisas nestas últimas duas semanas.

\begin{tabular}{|l|l|c|c|c|c|c|}
\hline & & Nada & $\begin{array}{c}\text { Muito } \\
\text { pouco }\end{array}$ & Médio & Muito & Completamente \\
\hline 10 & Você tem energia o suficiente para seu dia a dia? & 1 & 2 & 3 & 4 & 5 \\
\hline 11 & Você é capaz de aceitar sua aparência física? & 1 & 2 & 3 & 4 \\
\hline 12 & $\begin{array}{l}\text { Você tem dinheiro suficiente para satisfazer suas } \\
\text { necessidades? }\end{array}$ & 1 & 2 & 3 & 5 \\
\hline 13 & $\begin{array}{l}\text { Quão disponíveis para você estão as informações } \\
\text { que precisa no seu dia a dia? }\end{array}$ & & & & \\
\hline 14 & $\begin{array}{l}\text { Em que medida você tem oportunidade de ativi- } \\
\text { dade de lazer? }\end{array}$ & & & & \\
\hline
\end{tabular}

As questões seguintes perguntam sobre quão bem ou satisfeito você se sentiu a respeito de vários aspectos de sua vida nas últimas duas semanas.

\begin{tabular}{|c|c|c|c|c|c|c|}
\hline & & Muito ruim & Ruim & $\begin{array}{l}\text { Nem ruim nem } \\
\text { bom }\end{array}$ & Bom & Muito bom \\
\hline 15 & Quão bem você é capaz de se locomover? & 1 & 2 & 3 & 4 & 5 \\
\hline 16 & Quão satisfeito (a) você está com o seu sono? & 1 & 2 & 3 & 4 & 5 \\
\hline 18 & $\begin{array}{l}\text { Quão satisfeito (a) você está com sua capacidade } \\
\text { para o trabalho? }\end{array}$ & 1 & 2 & 3 & 4 & 5 \\
\hline 19 & Quão satisfeito (a) você está consigo mesmo? & 1 & 2 & 3 & 4 & 5 \\
\hline 20 & $\begin{array}{l}\text { Quão satisfeito (a) você está com suas relações } \\
\text { pessoais (amigos, parentes, conhecidos, colegas)? }\end{array}$ & 1 & 2 & 3 & 4 & 5 \\
\hline 23 & $\begin{array}{l}\text { Quão satisfeito (a) você está com as condições do } \\
\text { local onde mora? }\end{array}$ & 1 & 2 & 3 & 4 & 5 \\
\hline 24 & $\begin{array}{l}\text { Quão satisfeito (a) você está com o seu acesso } \\
\text { aos serviços de saúde? }\end{array}$ & 1 & 2 & 3 & 4 & 5 \\
\hline 25 & $\begin{array}{l}\text { Quão satisfeito (a) você está com o seu meio de } \\
\text { transporte? }\end{array}$ & 1 & 2 & 3 & 4 & 5 \\
\hline \multicolumn{7}{|c|}{ As questões seguintes referem-se a com que frequência você sentiu ou experimentou certas coisas nas últimas duas semanas. } \\
\hline & & Nunca & $\begin{array}{l}\text { Algumas } \\
\text { vezes }\end{array}$ & Frequentemente & $\begin{array}{l}\text { Muito } \\
\text { frequentemente }\end{array}$ & Sempre \\
\hline
\end{tabular}

Alguém Ihe ajudou a preencher este questionário?

Quanto tempo você levou para preencher este questionário?

Você tem algum comentário sobre o questionário?

Obrigada pela sua colaboração. 